\title{
Leitbild Nord-Süd : eine Bilanz der Umsetzung des Leitbilds in der Bundesverwaltung fünf Jahre nach seiner Verabschiedung
}

Xavier Tschumi and Catherine Schümperli Younossian

\section{CpenEdition}

Electronic version

URL: http://journals.openedition.org/sjep/766

DOI: $10.4000 /$ sjep.766

ISSN: 1663-9677

\section{Publisher}

Institut de hautes études internationales et du développement

Printed version

Date of publication: 1 avril 2000

Number of pages: 13-37

ISSN: 1660-5926

\section{Electronic reference}

Xavier Tschumi und Catherine Schümperli Younossian, „Leitbild Nord-Süd : eine Bilanz der Umsetzung des Leitbilds in der Bundesverwaltung fünf Jahre nach seiner Verabschiedung", Schweizerisches Jahrbuch für Entwicklungspolitik [Online], 19 | 2000, Online erschienen am: 21 Januar 2021, abgerufen am 11 März 2021. URL: http://journals.openedition.org/sjep/766 ; DOI: https://doi.org/10.4000/sjep. 766

This text was automatically generated on 11 mars 2021 .

(c) The Graduate Institute 


\title{
Leitbild Nord-Süd : eine Bilanz der Umsetzung des Leitbilds in der Bundesverwaltung fünf Jahre nach seiner Verabschiedung
}

\author{
Xavier Tschumi and Catherine Schümperli Younossian
}

\section{Einleitung}

1 Das Bewusstwerden der tiefgreifenden Veränderungen in der Welt und auf dem Planeten im Verlauf der 90er Jahre veranlasste die Schweiz dazu, ihre Aussenpolitik besonders mit den Entwicklungsländern - von Grand auf neu zu definieren. Der Bundesrat verabschiedete dazu zwei Berichte ${ }^{1}$, welche die Gesamtheit der Aussenbeziehungen der Schweiz umfassen. Weil beide Berichte dem Parlament unterbreitet und von diesem gebilligt wurden, genossen sie eine klare politische Unterstützung und stellten eine normative Richtung dar, welche die Schweiz einzuschlagen beschloss.

Der zweite Bericht, der besser unter dem Namen „Leitbild Nord-Süd” (Leitbild, LBNS) bekannt ist, behandelt insbesondere die Beziehungen der Schweiz (Norden) mit den Entwicklungsländern (Süden). Das Leitbild Nord-Süd geht über die reine Entwicklungszusammenarbeit der Schweiz hinaus, da es den Schwerpunkt auf die notwendige Artikulation der Gesamtheit der Beziehungen der Schweiz mit dem Süden legt: Wie wir im folgenden Kapitel sehen werden, drückt das LBNS in der Tat den klaren Willen aus, zu einem höheren Grad an Kohärenz zwischen den verschiedenen Politiken der Schweiz gegenüber den Entwicklungsländern zu gelangen. Es ist wirklich das Kohärenzkonzept, das dem Leitbild Nord-Süd und dadurch auch dem politischen Vorhaben der Schweiz für die 90er Jahre seine wahre Dimension verleiht.

Wie wurde dieses Vorhaben in der Praxis verwirklicht? In dieser Studie wollten wir fünf Jahre der Umsetzung des Leitbilds Nord-Süd in der Bundesverwaltung evaluieren ${ }^{2}$, 
besonders bei den Bundesämtern und Direktionen, die am unmittelbarsten in Verbindung mit den Entwicklungsländern arbeiten. Hierzu wurden fünf Bundesstellen wegen ihrer - auch indirekten, aber wichtigen - Rolle bei der Gestaltung und Umsetzung des Leitbilds identifiziert: die Direktion für Entwicklung und Zusammenarbeit (DEZA/EDA), das Staatssekretariat für Wirtschaft (seco/EVD), das Bundesamt für Umwelt, Wald und Landschaft (BUWAL/UVEK), das Bundesamt für Flüchtlinge (BFF/EJPD) und die Politische Abteilung IV, Menschenrechtspolitik und humanitäre Politik (PAIV/EDA). Diese Bundesämter und Direktionen bieten für unsere Studie den Vorteil, vier verschiedenen eidgenössischen Departementen anzugehören, zwischen denen die Kohärenz ihrer Politiken - zumindest nach dem Leitbild Nord-Süd - angestrebt und erreicht werden soll.

4 In Anbetracht des politisch heiklen Charakters unseres Themas und um über das Niveau des Wortlauts der Publikationen der Bundesverwaltung hinauszukommen, wollten wir mit den Personen sprechen, die sich aufgrund ihrer Position in jeder der fünf Dienststellen frei zur Frage äussern konnten, wenn sie es wünschten. Diese Gesprächspartner ${ }^{3}$ erklärten sich, nachdem sie unseren Entwurf erhalten hatten, zu einem teilgeleiteten Gespräch von durchschnittlich eineinhalb Stunden mit uns bereit. Wir möchten hier jedem von ihnen herzlich dafür danken, dass sie uns empfangen haben und uns ihre Meinung zum Leitbild Nord-Süd dargelegt haben. Die Zusammenfassung dieser Gespräche und der Lektüre der zusammengetragenen Unterlagen wird für jedes Bundesamt bzw. jede Direktion im Kapitel 3 vorgenommen.

Die Erörterung des dem Leitbild Nord-Süd zugrunde liegenden Kohärenzkonzepts erfordert, sich zumindest die Frage nach der Finalität der Kohärenz zu stellen ${ }^{4}$. Wofür und für wen soll Kohärenz angestrebt und erreicht werden? Um diese Fragen zu beantworten und unserer Studie eine Perspektive zu geben, schien es uns wichtig, zwei weitere Konzepte aufzugreifen, die eine grössere Kohärenz der öffentlichen Politiken fördern sollten und ebenfalls in den 90er Jahren ausgearbeitet wurden : das Konzept der „nachhaltigen Entwicklung” und das der „guten Regierungsführung”. Eine kurze Definition der beiden Konzepte und des ihnen eigenen Kohärenzverständnisses wird im folgenden Kapitel gegeben.

Im Kapitel kommen wir zum Abschluss auf die geteilte Verantwortung der schweizerischen politischen Behörden und der Bundesverwaltung zurück, was das Engagement der Schweiz für eine bessere Kohärenz ihrer öffentlichen Politiken gegenüber den Entwicklungsländern betrifft. In diesem Rahmen legen wir dar, wie die nachhaltige Entwicklung in der Schweiz im Verhältnis zum Leitbild Nord-Süd gehandhabt wurde.

\section{Historischer und konzeptueller rahmen}

\section{Entstehungsgeschichte und Inhalt des Leitbilds Nord-Süd}

Das LBNS wurde in einem geschichtlichen Umfeld ausgearbeitet, das man als im gesamten 20. Jahrhundert einzigartig bezeichnen kann. Nach Ansicht unserer Gesprachspartner konnten die besonderen Umstande, welche die Annahme des LBNS durch den Bundesrat ermöglicht haben - obwohl es erst fünf Jahre her ist - heute sicherlich nicht mehr gleichzeitig eintreten. 
8 Auf internationaler Ebene ist das Ende des Kalten Krieges zweifelsohne der entscheidende Faktor für den nahezu paradigmatischen Wechsel der internationalen Beziehungen: Ab 1989 nehmen die Beziehungen der Schweiz mit den Ländern des Südens und bald darauf auch mit den Oststaaten allmählich ein anderes Gesicht an. Daher sah sich die Schweiz veranlasst, über den neuen Inhalt dieser Beziehungen nachzudenken. Ebenfalls auf internationaler Ebene ist das Bewusstwerden der schwerwiegenden Gesundheitsprobleme des Planeten Erde ein weiterer entscheidender Faktor : Es müssten unverzüglich Fragen nach der Art von Entwicklung gestellt werden, für welche die Schweiz erst für sich selbst, dann in den Ländern des Südens eintreten wollte. Andere sogenannte globale Faktoren haben die Schweiz gleichfalls dazu veranlasst, ihre Aussenbeziehungen neu zu überdenken, wie zum Beispiel Fragen betreffend Migration, Drogen, Korruption oder die wirtschaftliche Globalisierung.

9 Auf nationaler Ebene ist der dem Leitbild Nord-Süd zugrunde liegende ausschlaggebende Faktor sicherlich der Wille der Schweiz, die Effizienz ihrer Entwicklungszusammenarbeit $\mathrm{zu}$ verstärken, in einem Kontext mit Budgetschwierigkeiten des Bundes und nach mehreren Jahrzehnten öffentlicher Hilfe mit insgesamt enttäuschenden Ergebnissen. Es ging darum, im Rahmen der internationalen Entwicklungszusammenarbeit mit weniger Mitteln die Dinge besser zu machen und mehr zu erreichen, umso mehr als die Zusammenarbeit sich auch auf einige Oststaaten auszudehnen begann, die sich vor kurzem von der Sowjetunion gelöst hatten.

10 Anhand all dieser Faktoren auf nationaler und internationaler Ebene haben die schweizerischen Behörden lange an der Neudefinition der Aussenbeziehungen ihres Landes gearbeitet. Ende 1993 verabschiedete der Bundesrat den Bericht über die Aussenpolitik der Schweiz in den 90er Jahren, von dem sich einige Monate später der Bericht über die Nord-Süd-Beziehungen derSchweiz (Leitbild Nord-Süd) ${ }^{5}$ leiten liess. Die Vorbereitung dieses Texts war von intensiven Verhandlungen zwischen den verschiedenen mit seiner Ausarbeitung beauftragten Bundesämtern und Direktionen geprägt - entsprechend den darin enthaltenen, potentiell auf dem Spiel stehenden Fragen, da von vornherein nicht ausgeschlossen war, dass dieser Text jeder Bundesstelle im Namen der Politikkohärenz ein wirkliches Recht auf Einmischung in die bis dahin (nahezu) privaten Angelegenheiten der anderen Dienststellen der Bundesverwaltung geben wurde.

11 Die beiden Berichte, die danach vom Parlament angenommen wurden, engagieren die Schweiz zur gleichzeitigen Verfolgung von vier strategischen Zielen ${ }^{6}$, welche die politischen, wirtschaftlichen, sozialen und ökologischen Aspekte der Aussenbeziehungen der Schweiz umfassen :

-politisches Ziel: Wahrung und Förderung von Frieden und Sicherheit, Förderung von Menschenrechten, Demokratie und Rechtsstaat ;

- wirtschaftliches Ziel : Förderung der Wohlfahrt ;

- soziales Ziel : Erhöhung der sozialen Gerechtigkeit ;

- ökologisches Ziel : Schutz der natürlichen Lebensgrundlagen.

Im Leitbild Nord-Süd ist jedes Ziel in eine Reihe von Aktionsfeldern unterteilt, die seine verschiedenen Aspekte veranschaulichen und der Arbeit der Bundesverwaltung, die mit der Umsetzung des LBNS beauftragt ist, eine etwas genauere normative Richtung geben. 
13 Die neue internationale und globale Konstellation, die den Ausgangspunkt des LBNS gebildet hatte, erforderte, dass all diese Ziele von der Schweiz als gleich wichtig angesehen werden. Davon ausgehend kommt man bei der Umsetzung des LBNS nicht um die schwierige Frage herum, wie sich bei der Verwirklichung eines Ziels Fortschritte erzielen lassen, ohne dabei bei der Verfolgung eines anderen Ziels in den Rückstand zu geraten, zumal die Politiken, die von verschiedenen Ämtern oder Direktionen der Bundesverwaltung gegenüber den Ländern des Südens durchgeführt werden, jeweils einem Ziel mehr Gewicht als einem anderen beimessen.

\section{Das Kohärenzkonzept}

14 Die obenerwähnte schwierige Frage findet im Kohärenzkonzept eine einfache theoretische Lösung. Das LBNS anerkennt, dass wenn die Politiken der verschiedenen Bundesstellen in Beziehung zu den Ländern des Südens kohärent sind, die vier von ihm festgelegten Ziele erreicht werden können. Bevor wir untersuchen, wie sich dies in der Realität niederschlägt, wenden wir uns der Bedeutung dieses Konzepts ${ }^{7} \mathrm{zu}$, das man auf drei Ebenen ins Auge fassen kann :

- die interne Kohärenz einer Politik, insbesondere der Politik der Entwicklungszusammenarbeit. Diese Kohärenzebene wird in der vorliegenden Studie nicht behandelt, da das Leitbild NordSüd anstrebt, die Gesamtheit der Beziehungen der Schweiz mit den Entwicklungsländern zu umfassen und nicht nur eine dieser Beziehungen (die Entwicklungszusammenarbeit), auch wenn diese im Nord-Süd-Kontext oft wichtig ist ;

- die Kohärenz zwischen den verschiedenen Politiken der Schweiz, die eine direkte Wirkung auf die Entwicklungsländer haben. Das LBNS legt besonderen Nachdruck auf diese Kohärenzebene im Hinblick auf die Harmonisierung der einzelnen Bereiche der schweizerischen Aussenpolitik gegenüber den Entwicklungsländern ;

- die Kohärenz zwischen der Aussen- und Innenpolitik der Schweiz. Das LBNS legt auch auf diese Kohärenzebene Nachdruck, da hier die Glaubwürdigkeit der Schweiz bei ihren Beziehungen mit den Ländern des Südens auf dem Spiel steht.

Folglich geht es im Leitbild Nord-Süd um die beiden letzteren Kohärenzebenen, und auch die Bundesverwaltung wird hierauf verwiesen. Die Bundesverwaltung, die mit der Umsetzung der Kohärenz auf diesen beiden Ebenen beauftragt ist, ist natürlich mit dem entscheidenden Punkt des gesamten Unterfangens („the acid test”) konfrontiert, zumal das Leitbild zu den konkreten Umsetzungsverfahren nichts aussagt. Bevor wir jedoch untersuchen, wie die Bundesstellen, die dem LBNS einen greifbaren Inhalt geben sollen, den Kohärenzbegriff verstanden haben, ist eine Bemerkung zum Begriff der Inkohärenz, des Misslingens der Kohärenz, erforderlich. Inkohärenz kann je nach den Bedingungen ihres Entstehens zwei Formen annehmen ${ }^{9}$ :

- ungewollte Inkohärenz, die dann entsteht, wenn zwei (oder mehrere) Bundesstellen ihre eigenen Politiken verfolgen, ohne miteinander einen Dialog zu führen, und diese Politiken sich bei näherer Betrachtung als inkohärent erweisen wurden. Das LBNS erlaubt es, diese Form von Inkohärenz ziemlich leicht zu vermeiden, da es der Bundesverwaltung zumindest das Streben nach der Kohärenz ihrer Politiken einflösst : Es genügt, Kohärenz anzustreben, damit ungewollte Inkohärenz nicht auftritt ;

- akzeptierte Inkohärenz (der Ausdruck „gewollte” Kohärenz wäre zu stark), die entsteht, wenn zwei (oder mehrere) Bundesstellen durch Verhandlungsverfahren erfolglos nach Kohärenz gestrebt haben. Diese Inkohärenz, die sich aus einem ungelösten Interessenkonflikt ergibt, 
der dann mittels eines politischen Beschlusses entschieden wird, wird somit in Ermangelung eines Besseren toleriert. Diese Form von Inkohärenz soll die Bundesverwaltung bei der Umsetzung des Leitbilds Nord-Süd soweit wie möglich vermeiden. Es genügt dabei nicht, Kohärenz anzustreben, sondern man muss sie auch erreichen.

\section{Das Konzept nachhaltiger Entwicklung}

16 Nachhaltige Entwicklung wird im Allgemeinen als eine Entwicklung definiert, „welche die heutigen Bedürfnisse $\mathrm{zu}$ decken vermag, ohne für künftige Generationen die Möglichkeit zu schmälern, ihre eigenen Bedürfnisse zu decken"10. Zu dieser Definition aus dem Jahre 1987 kommen noch die beiden folgenden Konzepte hinzu, die der nachhaltigen Entwicklung zugrunde liegen: „Das Konzept der 'Bedürfnisse', insbesondere der wesentlichen Bedürfnisse der Ärmsten, dem die grösste Priorität einzuräumen ist, und die Vorstellung von den Grenzen, die der Stand unserer Technik und unserer sozialen Organisation der Fähigkeit der Umwelt auferlegt, den

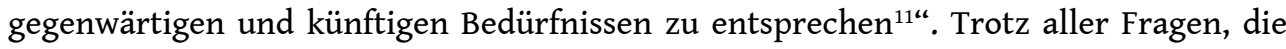
diese Definition aufwirft und auf die wir in dieser Studie nicht eingehen werden, gilt sie weiterhin als Referenz.

Infolge der Konferenz der Vereinten Nationen über Umwelt und Entwicklung (UNCED) - der Erdgipfel, der im Juni 1992 in Rio abgehalten wurde - hat das Konzept nachhaltiger Entwicklung einen gewaltigen Resonanzraum gefunden, da alle Länder, die an der Konferenz teilnahmen, darunter die Schweiz, sich verpflichteten, dem Konzept einen greifbaren Inhalt zu geben. In diesem Zusammenhang hat sich die Schweiz drei Hauptziele gesetzt, welche das LBNS praktisch unverändert übernommen hat : Umweltschutz, wirtschaftliche Effizienz und soziale Solidarität.

Um das Beiwort „nachhaltig” zu verdienen, macht es die nachhaltige Entwicklung zur Grundforderung, dass diese drei Ziele gleichzeitig verfolgt werden. Somit stellt sich die schwierige Frage, die oben in Bezug auf die Ziele des Leitbilds Nord-Süd identifiziert wurde, hier erneut und zwar folgendermassen : Wie kann man gleichzeitig in Richtung der drei Ziele vorankommen, ohne eines dieser Ziele zu vernachlässigen ? Wie bei der operationellen Umsetzung des LBNS stosst sich für die Schweiz die gleichzeitige praktische Umsetzung der drei Ziele nachhaltiger Entwicklung zwangsläufig an den Interessenkonflikten innerhalb der Bundesverwaltung selbst: Das Bundesamt oder die Direktion, die eines der Ziele nachhaltiger Entwicklung verfolgt, muss dies selbstverständlich weiterhin tun (weil das Ziel seiner/ihrer Spezialität bzw. seinen/ ihren Interessen entspricht), jedoch unter der Bedingung, dass andere Bundesstellen ähnliche Fortschritte in Richtung auf die beiden übrigen Ziele nachhaltiger Entwicklung machen. Unter dieser Bedingung ist es akzeptabel und vielleicht sogar förderlich, dass ein Ziel bei seiner Verwirklichung etwas rascher vorankommt als die beiden anderen Ziele, wegen der Synergien, die sich anschliessend daraus ergeben können. Jedoch darf kein Ziel bei der nachhaltigen Entwicklung übergangen werden. Deshalb muss die politische Entscheidung, die zur Lösung der unvermeidlichen Interessenkonflikte zwischen den Bundesstellen getroffen wird, ausgleichend wirken, indem sie einerseits die Politiken zur Umsetzung des am wenigsten fort-geschrittenen Ziels fördert und andererseits die Politiken zügelt, die zur Verwirklichung des am weitesten fortgeschrittenen Ziels beitragen. Dieses politische Ausbalancieren strebt somit eine gewisse Kohärenz bei der Verwirklichung der drei Ziele an, die sich die 
nachhaltige Entwicklung setzt; ohne diese Kohärenz gibt es schlichtweg keine nachhaltige Entwicklung.

\section{Das Konzept guter Regierrungsführung}

19 Gute Regierungsführung („Good Governance”) ist ein Konzept, das sich teilweise mit den Begriffen des politischen Ziels des Leitbilds Nord-Süd deckt: Rechtsstaat, Demokratie, Achtung der Menschenrechte, Korruptionsbekämpfung, angemessene Zuteilung öffentlicher Ressourcen, zivile Partizipation. Jedoch strebt gute Regierungsführung nicht selbst ein Ziel an, wie das Leitbild Nord-Süd und die nachhaltige Entwicklung dies tun: Seit Anfang der 90er Jahre wird gute Regierungsführung als eine Vorbedingung für jede Politik der Entwicklungszusammenarbeit und als Gewähr für die Effizienz dieser Zusammenarbeit betrachtet, da sie danach strebt, dem Land, dem die Zusammenarbeit zugute kommt, feste Grundlagen zu geben, auf denen sich die Entwicklung aufbauen kann. Und Kohärenz ist, wie wir gesehen haben, auch mit diesem Effizienzbegriff eng verbunden. In diesem Sinne erscheint gute Regierungsführung als Bedingung für die Kohärenz der öffentlichen Politiken, besonders im Rahmen der internationalen Entwicklungszusammenarbeit. Während die nachhaltige Entwicklung das politische Ziel des LBNS in sehr verschwommener Weise integriert, bringt die gute Regierungsführung hier eine nützliche Ergänzung dazu ein, auf die wir später (im Unterkapitel 3.5.) zurückkommen werden.

\section{Umsetzung des Leitbilds Nord-Süd durch de Schweizerische Bundesverwaltung}

Wir haben gesehen, dass das Leitbild Nord-Süd vor allem ein politisches Vorhaben ist, das die Reaktion der Schweiz auf die neue internationale und globale Konstellation widerspiegelt. Die vier Ziele, die das Leitbild sich setzt, tragen alle zur „langfristigen Wahrung der Schweizer Interessen" 12 bei. Das LBNS sieht vor, dass „kurz- und langfristige, nationale und internationale Interessen gegeneinander abzuwägen sind" ${ }^{13}$, ohne dass es darum geht, „Schweizer Interessen aufzugeben” ${ }^{14}$. Es ist beunruhigend, dass das LBNS sich mit dem axiomatischen Charakter des Begriffs des „gegeneinander Abwägens" der Interessen abzufinden scheint, da man sich, bevor man es erreicht, erst noch darüber einigen muss, was ein solches „gegeneinander Abwägen” ist, wenn man vermeiden will, dass jede der Bundesstellen sich eine recht unterschiedliche Vorstellung davon machen kann.

21 Folgt man den Ausführungen des Leitbilds, so läuft dieses "gegeneinander Abwägen" der Interessen innerhalb der Bundesverwaltung zwangsläufig auf die Kohärenz der öffentlichen Politiken hinaus. In der Tat gibt das LBNS stillschweigend $\mathrm{zu}$, dass ein "gegeneinander Abwägen" der Interessen sich in der Praxis der Bundesverwaltung in kohärenten Politiken niederschlagen wird. Dadurch wird die Verschwommenheit, die diesen Begriff „gegeneinander Abwägen” umgibt, auf das Kohärenzkonzept übertragen, wobei die Aufgabe der Bundesverwaltung, der es obliegt, das LBNS umzusetzen, erheblich erschwert wird.

Das Leitbild fordert wohl „eine kohärente Südpolitik” ${ }^{15}$, aber eine Politik, welche lediglich die Resultante eines Bündels von Politiken sein kann, die von Natur aus 
gegensätzlich sind. Denn das Kohärenzstreben ist nur bei einer Konfliktsituation zwischen unterschiedlichen Politiken notwendig, ob diese nun von verschiedenen Ämtern oder Direktionen der Bundesverwaltung verfolgt werden oder nicht. So strebt das Leitbild in erster Linie an, die Gegensätzlichkeiten zwischen den verschiedenen Politiken betreffend die Länder des Südens zu identifizieren und sie dann soweit wie möglich zu beseitigen. Ohne Gebrauchsanweisung zum Erreichen von Kohärenz wird jeder Politikkonflikt der Verhandlung und dem Kräfteverhältnis zwischen den beteiligten Bundesstellen überlassen.

In den folgenden Unterkapiteln werden wir sehen, dass jedes Bundesamt oder jede Direktion, die wir untersucht haben, vom Leitbild Nord-Süd einen persönlichen und variablen Gebrauch gemacht hat. Die Zusammenfassung des Gesprächs und der Unterlagen für jedes Amt bzw. jede Direktion geht im Allgemeinen einem analytischeren Teil unsererseits voraus. Wir zitieren keine wörtlichen Ausführungen aus den Gesprächen, sondern schliessen jedes Unterkapitel mit einer Definition der Kohärenz ab, die unsere Gesprächspartner uns in persönlicher Eigenschaft schriftlich zugehen liessen.

\section{Leitbild Nord-Süd}

Ziele - Aktionsfelder

1 - Wahrung und Förderung von Frieden und Sicherheit, Förderung von Menschenrechten, Demokratie und Rechtsstaat

211 - Gute Regierungsführung fördern

212 - Menschenrechte, Rechtsstaat und demokratische Prozesse durch positive Massnahmen fördern

213 - Zielkonflikte sichtbar machen*

214 - Rechtshilfe international ausgestalten

215 - Unverhältnismässige Militärausgaben in den Entwicklungsländern reduzieren

216 - Frieden schaffen und sichern

2 - Förderung der Wohlfahrt

221 - Die Rahmenbedingungen für ein nachhaltiges Wachstum der

Entwicklungsländer verbessern

222 - Den nichtstaatlichen Sektor in den Entwicklungsländern fördern

223 - Den Zugang für Produkte aus den Entwicklungsländern auf die Märkte des Nordens erleichtern

3 - Erhöhung der sozialen Gerechtigkeit

231 - Armut bekämpfen und soziale Gerechtigkeit fördern

232 - Das Bevölkerungswachstum reduzieren

233 - Eine kohärente und international koordinierte humanitäre Hilfe fördern

4 - Schutz der natürlichen Lebensgrundlagen

241 - In der Schweiz auf ein nachhaltiges Entwicklungsmodell hinarbeiten

242 - Der nachhaltigen Entwicklung international zum Durchbruch verhelfen 243 - Umwelt-, Handels- und Entwicklungspolitik in Einklang bringen 
244 - Wirtschafts-, Handels-, Aussen- und Entwicklungspolitik multilateral kohärent gestalten*

* Zwei Aktionsfelder des LBNS, welche die Kohärenz direkt betreffen, wurden im Aktionsplan der DEZA als „methodische Empfehlungen“ angesehen und waren fol $\mathrm{g}$ I ich nicht Gegenstand von Aktionslinien und spezifischen Massnahmen.

** $\mathrm{T}$ = Total, $\mathrm{E}$ = erfüllt, $\mathrm{W}$ = teilweise erfüllt, $\mathrm{N}$ = (noch) nicht erfüllt, $\mathrm{A}=$ ausgegliedert

\begin{tabular}{|c|c|c|c|c|c|c|}
\hline \multirow{2}{*}{ Aktionsplan } & \multirow{2}{*}{$\begin{array}{c}\text { Aktions } \\
\text { linien }\end{array}$} & \multicolumn{5}{|c|}{ Anzahl von Massnahmen** } \\
\hline & & $\mathbf{T}$ & $\mathbf{E}$ & w & $\mathbf{N}$ & A \\
\hline $\begin{array}{l}\text { - Wahrung und Förderung von Frieden und } \\
\text { Förderung von Menschenrechten, }\end{array}$ & 12 & 30 & 19 & 5 & $\Delta$ & 2 \\
\hline 2 - Förderung der Wohlfahrt & $\frac{12}{11}$ & 24 & $\frac{19}{13}$ & $\frac{5}{5}$ & 4 & 2 \\
\hline 3 -Erhöhung der sozialen Gerechtigkeit & 15 & 29 & 17 & 5 & 5 & 2 \\
\hline 4 -Schutz der natürlichen Lebensgrundlagen & 9 & 26 & 18 & 7 & 1 & 0 \\
\hline Total & 47 & 109 & 67 & 22 & 14 & 6 \\
\hline Durchschnittliche Erfüllung in Prozent & & & 62 & 20 & 13 & 5 \\
\hline
\end{tabular}

Quellen : Schweizerischer Bundesrat, Bericht des Bundesrates über die Nord-SüdBeziehungen...

Aktionsplan zum Leitbild Nord-Süd 1995-1998. Berichterstattung zur Umsetzung auf Ende 98, 5. Februar 1999 (interne DEZA-Unterlage).

\section{Direktion für Entwicklung und Zusammenarbeit (DEZA)}

Zur Durchführung des Leitbilds Nord-Süd hat die DEZA einen Aktionsplan in Form einer operationellen Umsetzung der vier strategischen Ziele und der 16 Aktionsfelder des Leitbilds ausgearbeitet ${ }^{16}$, der zur Identifikation von Aktionslinien führte, von denen jede in einem kurzen Erläuterungstext begründet wird. Jedoch liess es die DEZA nicht dabei bewenden, sondern unterteilte diese Aktionslinien noch in 109 spezifische Massnahmen. Mit jeder dieser Massnahmen sind die Namen der bei der DEZA für den Fortschritt der Massnahme verantwortlichen Mitarbeiter angegeben, wie auch deren eventuelle Partner in anderen Ämtern oder Direktionen der Bundesverwaltung und die Fristen, innerhalb derer jede spezifische Massnahme erfüllt werden muss.

Die DEZA erstellt mindestens einmal im Jahr eine Fortschrittsbilanz der Massnahmen ihres Aktionsplans, über die der nachstehende Rahmentext Aufschluss gibt. Die Aufstellung führt für jedes Ziel die Anzahl der erfüllten, teilweise erfüllten, (noch) nicht erfüllten oder ausgegliederten Massnahmen an. Die durchschnittliche Verwirklichung der Ziele, die am Ende der Aufstellung aufgeführt ist, gibt eine ziemlich genaue Vorstellung vom Erfüllungsgrad eines jeden Zieles, da keines von ihnen im Aktionsplan der DEZA wirklich vernachlässigt wurde. Lediglich das vierte Ziel ${ }^{17}$, bei dem im Übrigen keine Massnahme aufgegeben wurde, scheint vielleicht etwas weiter fortgeschritten zu sein.

Ferner sieht der Aktionsplan die Einsetzung interner Arbeitsgruppen in der Bundesverwaltung zur Umsetzung bestimmter, nach Themen zusammengefasster Massnahmen vor, insbesondere, um bei der Behandlung der verschiedenen Sondermassnahmen eine Synergie zu erreichen ${ }^{18}$. 

geringen Aussichten, verwirklicht zu werden, im Laufe der Zeit ausgegliedert wurden (eine als bescheiden erachtete Zahl). Jedoch sei vermerkt, dass eine dieser Massnahmen, welche aufgegeben wurde, weil man sie in der gegenwärtigen politischen Lage für unmöglich $\mathrm{zu}$ verwirklichen hielt, darauf abzielte, den Finanzplan dahingehend zu erstellen, die öffentliche Entwicklungshilfe in den ärmsten Ländern (LDC) bis zum Jahr 2000 auf 0,15\% des BSP anzuheben ${ }^{19}$. Die Aufgabe dieser Massnahme ist natürlich mit der Stagnation des Anteils, den die Schweiz ihrer gesamten internationalen Entwicklungszusammenarbeit zugewiesen hat, und ihrer immer offenkundigeren Unfähigkeit in Verbindung zu bringen, diesen Anteil auf 0,4\% des BSP (erklärtes Ziel des Bundesrates) anzuheben. Schwerpunktthema gemacht hatte, hat sie das LBNS so oft wie möglich dazu benutzt, die Diskussion innerhalb der Bundesverwaltung im Namen der Politikkohärenz mit Nachdruck zu betreiben. Gemäss der DEZA hat das LBNS es auf diese Weise verschiedenen Ämtern und Direktionen der Bundesverwaltung erlaubt, ihre Meinung $\mathrm{zu}$ äussern, um somit eine reichhaltige Grundlage für die darauf folgende politische Entscheidung auf der Ebene der eidgenössischen Departemente (selten des Bundesrates) zu beschaffen.

Die DEZA ist der Ansicht, dass das LBNS mehr Klarheit über die bei jeder politischen Entscheidung auf dem Spiel stehenden Fragen - selbst bescheidenster Art - erbringt. Für die DEZA gehört das LBNS von nun an zum Beschlussfassungssystem in der Bundesverwaltung, auch wenn ihre Beamten dies manchmal zu vergessen scheinen. Nachdem dies gesagt ist, räumt die DEZA die relative Bedeutung des LBNS für die Bundesinstanzen ein, die von Fragen betreffend die Entwicklungsländer etwas weiter entfernt sind und bei ihren politischen Beschlussfassungen andere Gesetze (oder einfach ihre politische Agenda) einhalten müssen. In dieser Situation fungiert die DEZA klar als die treibende Kraft für die Umsetzung des LBNS, mit dem Ziel, dem Kohärenzkonzept einen greifbaren Inhalt zu geben.

Aber auch die DEZA scheint schliesslich, fünf Jahre nach der Verabschiedung des Leitbilds Nord-Süd, von ihrer ursprünglichen Begeisterung etwas abgekommen zu sein. Ihr Aktionsplan, der zwischen 1995 und 1998 erfüllt werden sollte, befindet sich 1999 immer noch in Bearbeitung. Sogar das Wesen des Aktionsplans scheint sich geändert zu haben ; dieser Plan, der bei seiner Ausarbeitung die Umsetzung des LBNS anstrebte und der 1999 eher als ein strategisches Führungsinstrument erscheint, von dem sich die Jahresziele der DEZA (die ihrerseits wirklich operationell sind) leiten lassen würden ${ }^{20}$. Und dann ist es die DEZA ein wenig leid, dass die Kohärenz der öffentlichen Politiken der Schweiz (in ihren Augen) selten erreicht wird, umso mehr vielleicht, als diese Politikkohärenz seit der Verabschiedung des LBNS in der Bundesverwaltung immer öfter angestrebt wird. Es ist für die DEZA auf die Dauer frustrierend, festzustellen, dass die Bundesverwaltung sehr oft lediglich eine ungewollte Inkohärenz erreicht.

31 Schliesslich wurde auf die Initiative der DEZA eine Fassung des Leitbilds Nord-Süd für die allgemeine öffentlichkeit verbreitet (die allein von der DEZA herausgegeben wurde), um diesen Text einem grösseren Personenkreis als jenem, der das „Bundesblatt” einsieht, bekannt zu machen: „Eine Umsetzung dieser Ziele (des LBNS) ist weder denkbar noch machbar, ohne dass diese Bemühungen auch von der Bevölkerung mitgetragen werden" ${ }^{21}$. Namentlich weil sich die DEZA diese Behauptung

Schweizerisches Jahrbuch für Entwicklungspolitik, 19 | 2000 
zu eigen gemacht hat, ist das LBNS von der Aussenwelt nach und nach als "die Sache” der DEZA wahrgenommen worden, auch wenn letztere dies abstreitet: Das Leitbild Nord-Süd ist wirklich „eine Sache” des Bundesrates, unter anderen Sachen.

Der in diesen Aktionsplan investierte Arbeitsaufwand beweist unseres Erachtens dennoch, wie sehr die DEZA an diesem Leitbild hing und es vor allem zu einem Instrument machen wollte, auf das sie ihre Argumente zugunsten einer besseren Berücksichtigung der Interessen des Südens in der Bundesverwaltung stützen konnte. Man muss in dieser Hinsicht die Bemühungen der DEZA begrüssen. Jedoch finden wir in dem Aktionsplan keine wahrhaften Verfahren, die das Erreichen der Kohärenz ermöglichen; unserer Meinung nach bildet der Aktionsplan noch kein wirklich operationelles Dokument, umso weniger, als (fast) alle Massnahmen des Aktionsplans als „von oberster Priorität” eingestuft werden.

Man kann zwei Kohärenzebenen ins Auge fassen. Die erste, negative, ist das Fehlen von Inkohärenz, die zweite, positive, diejenige gegenseitiger Stärkung. Definieren wir zuerst einmal die Inkohärenz : Situation, die durch die gegenseitige Aufhebung von zwei Massnahmen verursacht wird. Beispiel : Die Schweiz beabsichtigt, die Korruption durch ihre Politik der Entwicklungszusammenarbeit zu bekämpfen, fördert sie aber tatsächlich durch ihre Steuergesetzgebung, die es gestattet, die für Bestechung gezahlten Summen vom steuerpflichtigen Einkommen abzuziehen. Die höhere, oder positive Kohärenzebene lauft darauf hinaus, alle zur Verfugung stehenden Instrumente zum gleichen Ziel einzusetzen.

Jean-François Giovannini, Stellvertretender Direktor, DEZA, 10. November 1999

Der Begriff der Kohärenz wird im entwicklungspolitischen Sprachgebrauch unterschiedlich verwendet. So bringt der Begriff zum Ausdruck, inwieweit verschiedene nationale Politiken eines Landes - welche direkte Wirkung in unseren Partnerländern zeigen - untereinander in Einklang, bzw. in Widerspruch stehen. Inkohärenzen sind folglich durch Zielkonflikte gekennzeichnet, die sich zwischen verschiedenen Politiken ergeben können. Das Bild „mit der einen Hand geben, mit der anderen Hand nehmen" bringt die Frage der Kohärenz auf den Punkt. Im Weiteren werden die (gegebenenfalls) unterschiedlichen Massstabe angesprochen, welche für gleichartige Fragestellungen in den Aussenbeziehungen, bzw. in der Innenpolitik zur Anwendung kommen. Mit der Kohärenz von Innenund Aussenpolitik steht die Glaubwürdigkeit eines Landes auf dem Spiel.

Marco Rossi, Chef der Sektion Politik und Forschung, DEZA, 15. November 1999

\section{Staatssekretariat für Wirtschaft (seco)}

Für das $\operatorname{seco}^{22}$ hat das Leitbild Nord-Süd eine andere Bedeutung als für die DEZA. Im Gegensatz zu anderen Bundesämtern wie BUWAL und DEZA verfolgt das seco nicht nur ein Ziel, sondern mehrere, indem es die Ziele von Wirtschaft, Entwicklung und Umwelt integrieren muss. Dies bedeutet, dass das seco die Kohärenzprobleme in der eigenen Behörde erlebt: Die Entscheidungen, die dort getroffen werden, erfordern häufig ein Abwägen zwischen Überlegungen betreffend die Entwicklung, die Erhaltung der Arbeitsplatze in der Schweiz, die Förderung der schweizerischen Wirtschafsinteressen im Ausland und anderen. Das seco besteht aus verschiedenen Direktionen, die sich mit Handel, Arbeitsmarkt, Wirtschaftsförderung und schliesslich mit der Zusammenarbeit 
mit den Entwicklungs- und Transitionsländern befassen (diese ist mit der DEZA für die Entwicklungspolitik mitverantwortlich). Das seco besitzt auch einen Umweltdienst.

Die Umsetzung des LBNS stellt für das seco eine komplexere Aufgabe dar als für die DEZA, da das seco sich nicht auf eine einfache Rolle als Fürsprecher der Entwicklungsländer beschränken kann. Aufgrund seiner vielfältigen Mandate muss das seco einen Ausgleichspunkt zwischen den kurz- und langfristigen Interessen der Schweizer Wirtschaft finden, wobei vorausgesetzt wird, dass die langfristige Perspektive für eine Position und eine operationelle Tätigkeit mit dem Ziel plädiert, den Entwicklungsländern einen bedeutenderen Platz in der Weltwirtschaft einzuräumen. Man kann sich im Übrigen vorstellen, dass das Unterfangen „Leitbild Nord-Süd" eine gewisse Zurückhaltung seitens des seco (damals BAWI) hervorgerufen hat, da dieses unter den Druck anderer Bundes-Ämter oder Direktionen geraten ist, die es zwingen, über gewisse Politiken $\mathrm{zu}$ verhandeln, die es vorher in nahezu selbständiger Weise verfolgte.

Nachdem dies gesagt ist, vertritt das seco die Ansicht, dass der Ausarbeitungsprozess des LBNS für das Staatssekretariat fast wichtiger war als seine darauffolgende Verabschiedung und die Bemühungen zu seiner Umsetzung. Das seco (seinerzeit BAWI) hat viel an der Abfassung des Leitbilds gearbeitet und hat in der langen Vorbereitungszeit dieses Dokuments (1990-1994) viel gelernt. Durch die intensive Mitwirkung an der Entstehung des LBNS war das seco zumindest zum Teil bestrebt, sicherzustellen, das der sich aus dieser übung ergebende Text keine Prioritäten festlegt, welche die wirtschaftlichen Realitäten ignorieren und/oder es nicht gestatten, die wirtschaftlichen Ziele der Schweiz zu verfolgen, oder die den Arbeitsplatz Schweiz im Rahmen der globalisierten Wirtschaft wettbewerbsunfähig machen. Nach Ansicht des seco sollte das LBNS ein Text bleiben, der eine politische Absicht in Bezug auf die Kohärenz zum Ausdruck bringt, und ein Instrument, das es erlaubt, offensichtliche Inkohärenzen zwischen Zielen klar zu identifizieren, ohne jedoch der Losung vorzugreifen oder eine Hierarchie zwischen den Zielen festzulegen. Das seco ist der Meinung, dass das LBNS auf keinen Fall eine Liste praktischer Modalitäten festlegen konnte, die in starrer Weise definieren, wie man zu dieser Kohärenz gelangt.

Demzufolge ist das seco, auch wenn die direkte Arbeit bei der Umsetzung des LBNS punktuell zu sein scheint, fast täglich mit dem Kohärenzproblem konfrontiert. Wie oben vermerkt, besteht die erste Priorität für das seco darin, die Kohärenz „innerhalb des Hauses", zwischen seiner Sektion für die wirtschaftliche Zusammenarbeit mit den Entwicklungs- und Transitionsländern und den übrigen Sektionen ${ }^{23} \mathrm{zu}$ suchen.

37 Anschliessend kann die Kohärenz, wie wir bereits erwähnten, nur das Ergebnis von Verhandlungen zwischen den Bundesämtern oder Direktionen von Fall zu Fall, je nach der Art der Frage und der auf dem Spiel stehenden Entscheidungen sein. Das Gleiche gilt, was die Beteiligung des seco an den zur Umsetzung des LBNS eingesetzten Arbeitsgruppen betrifft.

Schliesslich wäre es laut unserem Gesprächspartner falsch, zu glauben, dass das seco Entscheidungen nur gemäss den unmittelbaren Interessen der schweizerischen Privatwirtschaft trifft: Seit langem wird die umweltbezogene und die soziale Dimension berücksichtigt. Der Beschlussfassungsmechanismus innerhalb der schweizerischen Verwaltung zwingt dazu, eine Vielfalt von Interessen in Betracht $\mathrm{zu}$ ziehen; die Verabschiedung des LBNS hat diese Mechanismen zur Beratung und zur 
Abwägung der verschiedenen Interessen zweifelsohne verstärkt, auch wenn sie sicher nicht die einzige Ursache dafür ist.

Das seco erschien uns somit nuanciert zur Kohärenzfrage und beschränkt in den menschlichen Ressourcen, die es bereit ist, in eine lange Analysearbeit mit der DEZA und anderen Ämtern $\mathrm{zu}$ investieren, nicht weil es befürchten würde, dass seine Machtstellung in den Verhandlungen zwischen den verschiedenen Bundesstellen in Frage gestellt würde, sondern weil es sich ständig mit der Kohärenzproblematik auseinandersetzen muss aufgrund der unterschiedlichen Perspektiven, in denen seine Sektionen arbeiten. Fangt das Kohärenzstreben nicht für jedes Bundesamt oder jede Direktion mit der Kenntnis (bzw. der Erkennung und Anerkennung) der Strukturmerkmale der anderen Bundesstellen an?

Bei unserer wirtschaftlichen Zusammenarbeit mit den Entwicklungs- und Transitionsländern sind wir mit einem doppelten Kohärenzproblem konfrontiert. Das erste Problem bezieht sich auf das Gleichgewicht, das wir zwischen den verschiedenen Interessengruppen finden müssen, die unsere "Constituencies” bilden. Mit einer nicht ganz ernst gemeinten Bemerkung würde ich sagen, dass wir gute Chancen haben, kohärent zu sein, wenn der Vorort und die Hilfswerke beide mittelmässig unzufrieden sind mit dem, was wir machen!

Die zweite Dimension, auf die das Leitbild Nord-Süd nicht eingeht, die aber deshalb nicht minder wichtig ist, ist die Kohärenz zwischen unserem Beitrag und den Eigenanstrengungen der Empfängerländer. Es nützt nichts, dass die verschiedenen Aspekte unserer Zusammenarbeit mit einem Land untereinander kohärent sind, wenn sie nicht auch mit den Anstrengungen unserer Partner und anderer Geber kohärent sind.

Nicolas Imboden, Direktor des Leistungszentrums Entwicklung und Transition, seco, 14. Dezember 1999

\section{Bundesamt für Umwelt, Wald und Landschaft (BUWAL)}

Laut dem BUWAL entspricht die Förderung nach Kohärenz naturgemäss dem Querschnittscharakter der Umweltfragen (und noch mehr der Fragen nachhaltiger Entwicklung), mit denen es sich befasst. Diese Förderung ist im BUWAL tief verankert, da es sich bereits vor der Verabschiedung des Leitbilds Nord-Süd in diesem Sinne eingerichtet hat. Das LBNS wird daher vom BUWAL als eine Legitimation seiner Tätigkeit angesehen.

Doch mehr noch als das Leitbild Nord-Süd bildet das Konzept nachhaltiger Entwicklung den Bezugspunkt des BUWAL. Nachhaltige Entwicklung geht im Übrigen, nach Ansicht des BUWAL, mit demselben philosophischen Ansatz einher wie das LBNS. Das BUWAL unterstellt all seine Aktivitäten dem Geist nachhaltiger Entwicklung und misst daher dem Gedanken der Politikkohärenz hochrangige Bedeutung bei - welche die gleichzeitige Verwirklichung der drei (ökologischen, wirtschaftlichen und sozialen) Ziele anstrebt, ohne die es keine nachhaltige Entwicklung gibt. Unter diesem Gesichtspunkt kann das LBNS als eine Teileinheit oder Komponente der nachhaltigen Entwicklung betrachtet werden.

In seiner Strategie stellt das BUWAL seine Rolle als ,treibende Kraft in den Bemühungen, den Umweltschutz im Rahmen der nachhaltigen Entwicklung in die 
nationale und internationale Politik (der Schweiz) einzubeziehen" ${ }^{24}$ in den Vordergrund. Wir entnehmen aus diesem Leitgedanken, dass das BUWAL die nachhaltige Entwicklung etwas wie ein auf mehreren Pfeilern ruhendes Gebäude sieht, dessen Bau den am besten ausgestatteten Ämtern und Direktionen der Bundesverwaltung obliegen würde ${ }^{25}$. Der Umweltpfeiler dem BUWAL, der Wirtschaftspfeiler dem seco und der soziale Pfeiler der DEZA ? Warum nicht, aber unter der Bedingung, dass diese verschiedenen Arbeitsteams sich absprechen und sich bei ihrem jeweiligen Bauwerk gegenseitig helfen.

Auf dieser Ebene wurden die Beziehungen zwischen der Schweiz und den Entwicklungsländern vor der Konferenz von Rio 1992 fast ausschliesslich von der DEZA und vom seco (damals BAWI) verwaltet. Seitdem hat sich das BUWAL nach und nach, durch Dialog und Beratung mit diesen beiden traditionellen Akteuren der internationalen Entwicklungszusammenarbeit in diesen Bereich eingegliedert. Mit der DEZA hat das BUWAL sogar vor kurzem ein Vereinbarungsprotokoll unterzeichnet, das die Bedingungen ihrer Zusammenarbeit in den Schwerpunktländern der DEZA und für die dort vorgesehenen sektoriellen Strategien vorsieht. Die praktische Umsetzung dieses Abkommens, das (noch) nicht die Projekte der Entwicklungszusammenarbeit im eigentlichen Sinne umfasst, sollte in naher Zukunft beginnen. Mit dem seco sind die Fortschritte sehr viel langsamer, da es das seco nicht eilig zu haben scheint, ein ähnliches Protokoll mit dem BUWAL zu unterzeichnen. Jedoch nimmt das seco an den monatlichen Sitzungen der vom BUWAL geleiteten Arbeitsgruppe über Klimafragen teil.

Das BUWAL, dem die sektorübergreifende Arbeit zur zweiten Natur geworden ist, bedauert nichtsdestoweniger den Mangel an Dynamik, der innerhalb der Bundesverwaltung zur Frage der Politikkohärenz herrscht. Ferner bedauert das BUWAL, dass obwohl Synergien in der Verwaltung bestehen, diese sich zufällig ergeben, und dass die „Kultur” von Ämtern oder Direktionen immer noch sehr stark sei, trotz des Paradigmenwechsels ihrer Beziehungen zu den Ländern des Südens, die nicht mehr sektoriell angegangen werden können. Nach Meinung des BUWAL fehlt ganz offenbar ein Direktorium in der Bundesverwaltung, das fähig wäre, Synergien zwischen den Ämtern oder Direktionen, die mit dem Süden zu tun haben, zu nutzen oder entstehen zu lassen, und das auch eine gewisse Kohärenz gewährleisten könnte zwischen den Grundsätzen, die von der Schweiz in den multilateralen Foren vertreten werden, und den von ihr anschliessend erstellten bilateralen Politiken.

Wir fragen uns, ob die Entwicklungszusammenarbeit, die auf einem Gesetz, einem Beschluss und dem Leitbild Nord-Süd (sowie in geringerem Masse auf den Botschaften des Bundesrates zur Bewilligung von Rahmenkrediten) beruht, nicht zu einem neuen Gesetz umformuliert werden sollte, das den Süden und den Osten einschliesst, das aber vor allem das BUWAL formell und in gleichberechtigter Weise wie die DEZA und das seco umfasst. Dieser Vorschlag steht zweifellos nicht im Widerspruch zum Geist des Leitbilds Nord-Süd und könnte sicherlich eine bessere Kohärenz der öffentlichen Politiken der Schweiz gegenüber den Entwicklungs- oder Transitionsländern garantieren.

Das Streben nach Kohärenz ist ein Abstimmungsprozess, der es erlaubt, optimale Entscheidungen zu treffen, da sektorielle und lineare Entscheidungen zuweilen unwiederbringliche Schäden in anderen Bereichen hervorrufen können.

Kohärenz darf kein erstarrtes Idealkonzept sein. Sie setzt gegenseitige 
Beeinflussungen, Spannungen und Beschlüsse voraus, die nach einem Abwägen der Interessen endgültig entscheiden.

Philippe Roch, Direktor des BUWAL, 6. Dezember 1999

\section{Bundesamt für Flüchtlinge (BFF)} Aussenbeziehungen der Schweiz am besten ermisst. Denn diese Beziehungen, ob bilateral oder multilateral, ob mit Ländern des Südens, des Ostens oder des Nordens, haben einen Einfluss auf die Migrationsfrage, auch wenn der Einfluss im Fall der Schweiz minimal bleibt. Migrationen sind ein Ergebnis dieser Interpendenz der internationalen Beziehungen und somit auch der Kohärenz, mit der die Politiken, die diese Beziehungen anknüpfen, geführt werden.

Für das BFF, das direkt mit der Migrationsfrage konfrontiert ist, nimmt die Kohärenz der Aussenpolitik der Schweiz eine besondere Bedeutung an. Das BFF bedauert jedoch, dass das Leitbild Nord-Süd dieser Frage nicht die Bedeutung beimisst, die sie verdienen würde. Das Leitbild bleibt zu den internationalen Migrationserscheinungen vage, wohingegen diese Erscheinungen, wenn sie die Schweiz berühren, von der Schweizer Bevölkerung als das Problem Nummer eins angesehen werden. Die Gründe hierfür liegen vielleicht im hauptsachlich bilateralen und nach dem Süden (im engeren Sinne) ausgerichteten Charakter des Leitbilds, während die Migrationen ihrerseits eine Frage darstellen, die multilateral geregelt werden muss, und die Migrationen, welche die Schweiz betreffen, ihren Ursprung mehrheitlich in Europa selbst haben.

Wenn die Asylbewerber einmal auf schweizerischem Territorium angekommen sind, obliegt es dem BFF, die geeigneten Lösungen zu finden, was ihm oft die Rolle des Sozialhelfers und Polizisten einträgt. Die DEZA, mit der die Rolle des Krankenpflegers verbunden wird, befindet sich somit in Bezug auf die Migrationsfrage in einer etwas komfortableren Lage. Jedoch haben sich die Mentalitäten in diesen beiden Bundesstellen geändert; sie haben eine gewisse gegenseitige Annäherung eingeleitet, wobei die DEZA zum Beispiel bestimmten Herkunftsländern der Asylbewerber (besonders Albanien und Bosnien-Herzegowina) eine operationelle Priorität eingeräumt hat.

Für das BFF ist es rechtlich und moralisch gesehen klar, dass diese Länder ihre in der Schweiz weilenden Staatsbürger längerfristig wieder aufnehmen müssen, es sei denn, aussergewöhnliche humanitäre Gründe sprächen dagegen. Folglich muss die Schweiz ihre Entwicklungszusammenarbeit anpassen, wie die Europäische Union dies bereits getan hat: Der Kausalzusammenhang „Armut - Migrationen" wird immer mehr als Fiktion angesehen, während die Kettenreaktion „Nichteinhaltung der Menschenrechte - Staatsverfall - Migrationen" diese internationalen Bevölkerungsbewegungen besser zu erklären scheint. In diesem Sinne erweist sich das Leitbild Nord-Süd, zumindest in der Theorie als stark, da es der Entwicklungszusammenarbeit nicht mehr nur die wirtschaftliche und soziale Dimension beimisst. Die DEZA und das seco als die an der Entwicklungszusammenarbeit am meisten beteiligten Bundesstellen können für das BFF in ihren Schwerpunktländern über diese politischen Fragen wachen und dadurch der Kohärenz einen Punkt einbringen. Diese Annäherung zwischen der Aussen- und Innenpolitik der Schweiz wird auch von den schweizerischen NRO gefordert. 
Zwischen Ende 1995 und Anfang 1996 haben BFF, DEZA und seco (damals BAWI) unter der Schirmherrschaft der Beratenden Kommission für internationale Zusammenarbeit miteinander an der Migrationsfrage gearbeitet. Der aus dieser Annäherung hervorgegangene Bericht ${ }^{26}$ umfasst in 21 Thesen die wichtigsten Aspekte der Migrationen, die Zielkonflikte, die diese hervorbringen können sowie die möglichen Lösungen und Instrumente, welche die Schweiz zur Verhütung oder Behandlung dieser Frage ausarbeiten kann. Dieser Bericht über Migrationen und Entwicklungszusammenarbeit ist leider toter Buchstabe geblieben, weil er $\mathrm{zu}$ theoretisch und nach Meinung des BFF viel zu stark auf die Entwicklung ausgerichtet war, ohne wirkliche direkte Zusammenhänge mit der Migrationsfrage. Er versuchte gleichwohl, die Synergien und Zielkonflikte zwischen den Migrationen und der Entwicklungszusammenarbeit zu umfassen und stellte somit eine Bemühung für eine bessere Politikkohärenz dar. Jedoch scheint es, dass im Laufe des Jahres 2000 ein neuer, operationellerer Bericht von der Kommission diskutiert werden wird.

Die Schweiz ist bestrebt, bei der Formulierung und der Durchführung ihrer nationalen und internationalen Politik den Nutzen zu maximieren, den die Entwicklungsländer aus ihrem Austausch mit unserem Land ziehen können. Die Schweiz räumt offen Situationen ein, in denen nationale Interessen, die auf demokratische Weise formuliert werden oder sich aus der Aktion von Interessengruppen ergeben, Bestimmungen auferlegen, welche diese öffnung und diese Chancen tatsächlich und/oder von Rechts wegen mindern. Im Einwanderungs- und Asylbereich beschränkt sich die mögliche Kohärenz heute auf die Förderung der Menschenrechte und demokratischer Institutionen in der Schweiz wie im Ausland, auf die Anerkennung des individuellen Rechts auf Schutz bei politischer Verfolgung, die kollektive Aufnahme gefährdeter Personen und die Nichtdiskriminierung bei der Anwendung restriktiver Massnahmen beim Zugang zum Arbeitsmarkt. Die Anerkennung der Tatsache, dass in der Wirtschaft des Landes eine bedeutende und ungedeckte Nachfrage nach nicht qualifizierten Arbeitskräften besteht, könnte in diesem Zusammenhang bedeutende Verbesserungen erbringen.

Jörg Frieden, Chef des Bereichs der Direktion Finanzen und Soziales, BFF, 10. November 1999

\section{Politische Abteilung IV des eidgenössischen Départements für auswärtige Angelegenheiten (PAIV)}

51 Die PAIV ist die Abteilung, die beim EDA für Menschenrechtspolitik und humanitäre Politik zuständig ist. Ihr Aktionsrahmen wird hauptsächlich durch den Bericht des Bundesrates über die Menschenrechtspolitik von 1982 und den Bericht über die Aussenpolitik der Schweiz in den 90er Jahren von 1993 (vgl. Ziffer 412) bestimmt. Im Bereich der Beziehungen zwischen Menschenrechten und Entwicklungszusammenarbeit dient das Leitbild Nord-Süd des Bundesrates (1994), ergänzt durch die Leitlinien des EDA über die Förderung der Menschenrechte in der Entwicklungszusammenarbeit ${ }^{27}$, als Bezugsrahmen.

Was die Kohärenz der Politiken (Menschenrechts-, Entwicklungs-, Aussenwirtschafts-, Asylpolitik usw.) betrifft, so fordert die Politische Abteilung IV uns auf, die Antwort auf 
das Postulat Bäumlin (vom 17. Dezember 1997) zu verfolgen, die sie seit fast zwei Jahren vorbereitet. Das Postulat hat folgenden Wortlaut: „Der Bundesrat erstattet einen Kohärenzbericht zur Aussenpolitik der Schweiz in den neunziger Jahren, zum Menschenrechtsbericht von 1982, zu den OSZE-Aktivitäten der Schweiz (insbesondere im Bereich Minderheitenschutz), unter Einbezug aussenwirtschaftspolitischer und entwicklungspolitischer Aktivitäten (insbesondere unter dem Aspekt bilateraler und multilateraler „good governance guidelines").

Die verschiedenen involvierten Departemente und Bundesämter sind in die Berichterstattung so einzubeziehen, dass Divergenzen offengelegt und alsdann überwunden werden können. Die Federführung liegt beim EDA, namentlich bei der „Politischen Abteilung”.

Die Antwort auf dieses Postulat befindet sich seit vielen Monaten im Vernehmlassungsverfahren im EDA selbst, aber auch mit den Bundesstellen ausserhalb des EDA. Dieses Vernehmlassungsverfahren ist die typische Arena, in der die Interessen jeder Dienststelle im Sinne des Leitbilds Nord-Süd „gegeneinander abgewogen” werden müssen. Die Antwort auf das Postulat Bäumlin wird demnach das Ergebnis eines Konsenses sein, der zwischen allen Bundesstellen ausgehandelt wird, die an diesem Verfahren beteiligt sind.

Wenn diese Antwort Gegenstand solch schwieriger Verhandlungen ist, so kommt das vielleicht auch daher, dass sich die Antwort zu bestimmten Aspekten nicht mit Absichten zufrieden gibt, sondern die Einführung von Mechanismen anstrebt. Diese sollten in grossen Linien festlegen, wie man in einer gegebenen Art von Situation Fragen der Menschenrechte, der Demokratie und des Rechtsstaates mit anderen Fragen, zum Beispiel des Asylwesens, der Aussenwirtschaftspolitik oder der Entwicklungszusammenarbeit verbindet. Zum Aspekt der politischen Konditionalität, der unter diese Antwort fällt, wurden beispielsweise Mechanismen aufgeführt, die eine mögliche Unterbrechung der Entwicklungszusammenarbeit in einem Land festlegen, wenn die Menschenrechte dort missachtet werden. Dies verweist auf den im Postulat erwähnten Begriff der „good governance guidelines”, den wir im Kapitel 2 unter dem Ausdruck „gute Regierungsführung” behandelt haben.

Somit wird die Antwort auf das Postulat Bäumlin, wenn sie einmal bereit sein wird, um vom Bundesrat verabschiedet und dann an die eidgenössischen Rate weitergeleitet $\mathrm{zu}$ werden, das Resultat des Interessenspiels innerhalb der Bundesverwaltung sein. Nichtsdestoweniger wird man zwischen den Zeilen lesen können, wie diese Interessen ins Spiel gebracht wurden, welche Interessen in dem einen oder anderen Teil des Textes überwogen haben und welche sich nicht durchsetzen konnten, und ob diese Antwort schliesslich geeignet sein wird, gewissen öffentlichen Politiken der Schweiz eine grössere Kohärenz zu verleihen.

Das Engagement der Schweiz für Menschenrechte, Demokratie und Rechtsstaat ist einer der fünf Schwerpunkte der Aussenpolitik der Schweiz. Um glaubwürdig zu sein, muss unsere Aktion in diesem Bereich kohärent sein. Dies setzt voraus, dass unsere Politik sich durch eine umfassendere Berücksichtigung der Kriterien der Menschenrechte, der Demokratie und des Rechtsstaats bei jeder Entscheidung niederschlagen muss, die unter die allgemeine Politik der Eidgenossenschaft fallt. Dies muss ganz besonders in den Bereichen der Ausländerpolitik, der Asylpolitik, 
der Aussenwirtschaftspolitik und der Entwicklungspolitik der Fall sein.

Jean-Daniel Vigny, ministre, PAIV, 9. November 1999

\section{Kohärenz zwischen politischem vorhaben und tatsächlichem Inhalt}

Das vorhergehende Kapitel hat - falls nötig - gezeigt, dass die Umsetzung des Leitbilds Nord-Süd ohne klare politische Unterstutzung ein besonders schwieriges Unterfangen ist. Das Leitbild stellt sicherlich ein politisches Vorhaben dar, das darauf abzielt, mehr Kohärenz in die Gesamtheit der Beziehungen der Schweiz mit den Entwicklungsländern $\mathrm{zu}$ bringen. Es drückt den Willen der obersten politischen Behörden der Schweiz in dieser Sache aus. Jedoch muss dazu noch den Bundesstellen, die mit der operationellen Umsetzung des Leitbilds beauftragt sind, Orientierungshilfe bezüglich der Bedeutung gegeben werden ; wie soll man zum Beispiel eine Formulierung wie das ,gegeneinander Abwägen" der Interessen verstehen, die sich leicht zu vielfältigen Auslegungen eignet?

Tatsächlich haben die vom LBNS und von der Politikkohärenz betroffenen Bundesstellen nichts anderes getan, als jeweils von Fall zu Fall ihre eigenen Argumente darzulegen, um bei der darauf folgenden politischen Entscheidung eine möglichst breite Palette auf dem Spiel stehender Fragen einzubringen. Bisher wird die Kohärenz von den Bundesstellen lediglich angestrebt, wobei diese ihre Argumente in eine Art Gemeinschaftskasse einbringen, die zu diesem Anlass eingerichtet wurde. Zumindest wird die ungewollte Inkohärenz vermieden.

Auf der Grundlage all dieser Argumente muss eine politische Entscheidung getroffen werden, von welcher der Erfolg der Kohärenz - oder das Weiterbestehen der Inkohärenz, die leider in Ermangelung eines Besseren akzeptiert wird - abhängt. Aus den Gesprächen, die wir in den fünf Bundesstellen geführt haben, welche gegenüber den Entwicklungsländern am meisten engagiert sind, ergab sich, dass diese politische Entscheidung in der Mehrheit der Fälle zwischen den Departementen getroffen werden muss, denen diese Dienststellen angehören. Das scheint uns ein Problem des „Richtens in eigener Sache" aufzuwerfen: Wie sollten diese Departemente eine die Kohärenz gewährleistende politische Entscheidung fällen, während sie in der Verhandlung, die diese Entscheidung lenkt, lediglich ihre Interessen vertreten? Gibt es niemand, um darüber zu wachen, dass diese - theoretisch gewollte - Kohärenz durch ausgleichende Verfahren statt durch das Gesetz des Stärkeren einen praktischen Inhalt findet ? Soll die unsichtbare Hand der Bundesverwaltung die Kohärenzfrage nach dem Beispiel der unsichtbaren Hand des Marktes regeln? Das Leitbild Nord-Süd hat sich vier gleichbedeutende Ziele gesetzt, wohingegen die Departemente, die hinter diesen Zielen stehen und sie fördern, ihrerseits nicht gleich stark sind. Ist dies wirklich, was die obersten politischen Behörden der Schweiz gewollt haben?

„Aussenpolitische Kohärenz kann - in dem Masse wie sie politisch als wünschenswert erachtet wird - nur durch entsprechende Verfahren erhöht werden" ${ }^{28}$ : Das in der Bundesverwaltung bestehende Kollegialitätssystem würde es erlauben, den Willen, kohärent $\mathrm{zu}$ sein, leicht in Kohärenz $\mathrm{zu}$ verwandeln. Zudem könnte sich die Bundesregierung einrichten, indem sie einen grösseren Anteil ihrer menschlichen Ressourcen für die Kohärenzfrage einsetzt, sich zu diesem Zweck „rechtlich verbindliche Konsultationsmechanismen" ${ }^{29}$ gibt und gegebenenfalls eine 
Kohärenzkontrolle einrichtet. Daher ist es bedauerlich, dass es unter diesen Umständen so sehr am politischen Willen fehlt, der Politikkohärenz einen greifbaren Inhalt zu geben.

Obwohl das Leitbild Nord-Süd ein politisches Vorhaben ist, hat es keinen klaren politischen Willen $\mathrm{zu}$ seiner Umsetzung hervorgebracht. Die institutionellen Strukturen, die infolge dieser Umsetzung entstanden sind - und alsbald abgebaut wurden - sind dem oben beschriebenen Gesetz des Stärkeren nicht entgangen, wobei sie ihre Arbeiten mit Publikationen abschlossen, die insgesamt nicht immer hinreichend operationell waren.

Zum Vergleich ist die Umsetzung der nachhaltigen Entwicklung sehr aufschlussreich : Einer der ersten Schritte der Schweiz in Richtung auf die nachhaltige Entwicklung war die Einsetzung des Interdepartementalen Ausschusses Rio (IDA-Rio) im März 1993. Der IDA-Rio umfasst 20 Bundesämter, die beauftragt wurden, „eine Bestandesaufnahme über die Umsetzung der nachhaltigen Entwicklung in der Schweiz im Rahmen der sektoriellen Politiken und der Koordinationsmechanismen zu erarbeiten" ${ }^{30}$. Diese formelle interdepartementale Struktur ist aus einem echten politischen Willen hervorgegangen ; es ist jedoch unleugbar, dass für das Leitbild Nord-Süd ein ähnlicher Wille nicht aufgebracht wurde. Zwar hat sich die Schweiz international verpflichtet, die nachhaltige Entwicklung umzusetzen, woraus sich dieser politische gute Wille vielleicht erklärt. Doch gerät die im LBNS erwähnte Kohärenz anlässlich der alle vier Jahre erfolgenden Prüfung der internationalen Entwicklungszusammenarbeit durch die Kollegen der OECD ebenfalls ins internationale Visier. Gewiss wird die internationale Entwicklungszusammenarbeit der Schweiz von der OECD fast ständig als ein zu befolgendes Beispiel betrachtet, und die Empfehlungen, die diese Organisation formulieren könnte, sind im Grossen und Ganzen nicht sehr zwingend. Ist dies bei der nachhaltigen Entwicklung anders? Man kann sich zu Recht fragen, ob die Kommissionen für nachhaltige Entwicklung, die auf multilateraler Ebene regelmässig den Stand des Fortschritts bei der Umsetzung der nachhaltigen Entwicklung in jedem Land, auch in der Schweiz, ermitteln, nicht die wirkliche Lage verfälschen, indem sie ein etwas abgeschwächtes Bild davon geben.

Wir finden schliesslich wenig Grande dafür, warum das Leitbild Nord-Süd bei seiner Umsetzung nicht mehr politische Unterstützung erhalten hat. Gewiss gestatten die Zeit-, Personal- und Geldmittel es der Bundesverwaltung nicht, eine Vielzahl formeller Strukturen innerhalb der Verwaltung $\mathrm{zu}$ errichten. Im Anschluss an die Verabschiedung des LBNS war es wahrscheinlich überflüssig, nach der Struktur für nachhaltige Entwicklung eine neue interdepartementale Struktur einzusetzen, zumal die Ziele der nachhaltigen Entwicklung praktisch unverändert vom Leitbild Nord-Süd übernommen wurden und die von der nachhaltigen Entwicklung gewollte Kohärenz noch umfassender zu sein scheint als die des LBNS. Wir stellen hier die Hypothese auf, dass wenn das Leitbild Nord-Süd als solches zustande kommen musste, da es im Bericht über die Aussenpolitik der Schweiz. Ende 1993 angekündigt wurde, seine Umsetzung sich etwas mit den in Rio zur nachhaltigen Entwicklung eingegangenen Verpflichtungen überschnitten hat, deren operationelle Umsetzung unverzüglich, das heisst, ein Jahr vor der Verabschiedung des LBNS, begonnen hatte.

Das LBNS ist gleichwohl hinsichtlich seiner Stellung gegenüber der nachhaltigen Entwicklung nicht klar, wie zum Beispiel seine Aktionsfelder 221 und 241 bezeugen : Sie vermitteln die Vorstellung, dass sie die nachhaltige Entwicklung integrieren, während 
unserer Ansicht nach eher das Gegenteil der Fall wäre: Die vom LBNS angestrebte Kohärenz ist ein grundlegender Aspekt der nachhaltigen Entwicklung.

Wie dem auch sei, das Leitbild Nord-Süd und die Kohärenz, die es fördern will, sind der Bundesverwaltung überlassen, während sie auf die eine oder andere Weise in die interdepartementale Struktur eingegliedert hätten werden können, welche geschaffen wurde, um der nachhaltigen Entwicklung einen konkreten Inhalt zu geben. Doch seit fünf Jahren ist das Kohärenzstreben ein im Gang befindlicher Prozess, und der mehr oder minder gewundene Verlauf, den dieses Streben annehmen wird, hangt von den Bemühungen zur Förderung seiner Verwirklichung und von den sich dagegen erhebenden Widerstanden ab. Wenn die Schweiz dieses Kräfteverhältnis zugunsten einer besseren Kohärenz der öffentlichen Politiken nicht in formeller Weise ausgleichen will, wird die vorliegende Studie möglicherweise dazu beitragen, sie daran zu erinnern, dass das Leitbild Nord-Süd und die nachhaltige Entwicklung, die sie akzeptiert hat, sie ermahnen, so weit wie möglich darüber zu wachen. Die „Wache” existiert bereits in verschwommener Form in den Kreisen der NRO und der Universitätsinstitutionen. Sie könnte sich noch weiter organisieren, beispielsweise in Form einer Einrichtung zur Überwachung der Kohärenz, um sowohl die (sich noch ergebenden) ungewollten Inkohärenzen als auch die akzeptierten Inkohärenzen so systematisch wie möglich ans Licht zu bringen.

Es scheint schliesslich, dass das Leitbild Nord-Süd bei seiner operationellen Umsetzung innerhalb der Bundesverwaltung selbst, aber auch bei den verwaltungsexternen Akteuren, denen die Qualität der Nord-Süd-Beziehungen der Schweiz ein Anliegen ist, grosse Frustration hervorruft. Das LBNS hatte seinerzeit grosse Hoffnung geweckt. Es sollte das Unbehagen beheben, das die NRO sehr früh mit der Formel „mit der einen Hand geben, mit der anderen Hand nehmen" herausgestellt hatten. Im Umfeld zunehmender Wechselbeziehungen zwischen den Ländern und zwischen der Aussenpolitik der Länder, das die 90er Jahre kennzeichnet, konnte das Leitbild NordSüd jedoch die qualitative Veränderung der Beziehungen der Schweiz mit den Entwicklungsländern nicht allein vollziehen. Deshalb greifen wir hier die Frage wieder auf, die im Unterkapitel über das BUWAL (3.3.) aufgeworfen wurde: Sollte die Bundesverwaltung nicht erwägen, ein neues Gesetz über die internationale Entwicklungszusammenarbeit abzufassen, zumal der Bundesbeschluss über die Zusammenarbeit mit den Staaten Osteuropas im Jahr 2008 ausläuft? Nicht, dass die unzusammenhängenden gesetzgebenden Elemente, die der internationalen Entwicklungszusammenarbeit der Schweiz zugrunde liegen, nicht mehr angepasst seien - aber um dieser Zusammenarbeit ein einziges Fundament zu geben, das dauerhaft ist und die jüngste Entwicklung der Nord-Süd-Beziehungen berücksichtigt. Diese Dauerhaftigkeit wäre in unseren Augen die Gewahr für eine bessere Kohärenz der öffentlichen schweizerischen Politiken.

Somit können wir abschliessend feststellen, dass das Leitbild Nord-Süd sicherlich ein Stein in dem noch im Bau befindlichen Kohärenzgebäude ist. Die Verpflichtungen der Schweiz zugunsten der nachhaltigen Entwicklung sind ein weiterer Stein. Aus dieser Sicht geht es nicht so sehr darum, die Steine als solche zu analysieren, sondern die Geschwindigkeit, mit der sie aufeinandergesetzt werden, zu untersuchen. Hierbei nimmt der Plan des Gebäudes eine wesentliche Bedeutung an - doch muss er erst noch weitgehend entworfen werden. 


\begin{tabular}{|c|c|c|c|c|}
\hline \multicolumn{2}{|c|}{ BEISPIELE } & \multicolumn{2}{|c|}{$\begin{array}{l}\text { IN VoRBEREITUNG BEFINDLICHE } \\
\text { ODER VERABSCHIEDETE MASSNAHMEN }\end{array}$} & \multirow{2}{*}{$\begin{array}{l}\text { BEURTEILUNG DER POLITIKKOHÄRENZ } \\
\text { VOM ENTWICKLUNGSSTANDPUNKT } \\
\text { (SEIT 1994) } \\
\rtimes \text { VERBESSERUNG } \searrow \text { RÜCKGANG } \\
\rightarrow \text { UNVERÄNDERTER ZUSTAND }\end{array}$} \\
\hline $\begin{array}{l}\text { DAs LeTIBILd NoRd-SüD } \\
\text { ZIEIT DARAUF AB: }\end{array}$ & $\begin{array}{l}\text { Das LeITBILD NoRd-Süd } \\
\text { KANN KoNFIKTE IN FOLGENDEN } \\
\text { BEREICHEN DEUTLCH MACHEN: }\end{array}$ & $\begin{array}{l}\text { AUF BUNDESVERWALTUNGS- } \\
\text { EBENE GETROFFENE } \\
\text { MASSNAHMEN }\end{array}$ & $\begin{array}{l}\text { GeserzGeBunGs- } \\
\text { MASSNAHMEN }\end{array}$ & \\
\hline $\begin{array}{l}\text { Unverhältnismässige Militär- } \\
\text { ausgaben in den Entwick- } \\
\text { lungsländern zu reduzieren. }\end{array}$ & $\begin{array}{l}\text { Ausfuhr von Waffen oder } \\
\text { von Material, das für } \\
\text { militärischen Gebrauch } \\
\text { dienen kann, aus der } \\
\text { Schweiz in die Entwick- } \\
\text { lungsländer. }\end{array}$ & $\begin{array}{l}\text { Die DEZA hat eine Krite- } \\
\text { rienliste erstellt, die es } \\
\text { ihr gestattet, ihre Mei- } \\
\text { nung zu Waffenexporten } \\
\text { zu äussern. } \\
\text { (Neue Kriegsmaterialge- } \\
\text { setzgebung und neue } \\
\text { Arbeitshilfe für DEZA- } \\
\text { Mitarbeiterlnnen, 30.3.98) }\end{array}$ & $\begin{array}{l}\text { Revision des Bundesge- } \\
\text { setzes über das Kriegs- } \\
\text { material - angenommen } \\
\text { am 13.12.1996. } \\
\text { Güterkontrollgesetz über } \\
\text { Dual-use-Güter. } \\
\text { @ JsDW 1997, S. 109-114 }\end{array}$ & $\begin{array}{l}\rightarrow \text { Der Gesetzesentwurf des Bundesrates } \\
\text { sah vor, im Gesetz die Notwendigkeit fest- } \\
\text { zuschreiben, die Lage der Mensschenrechte } \\
\text { oder das Engagement der Schweiz im } \\
\text { Bestimmungsland zu berücksichtigen. Diese } \\
\text { Bestimmung wurde von den eidgenössi- } \\
\text { schen Räten bei der Verabschiedung des } \\
\text { Gesetzes nicht festgehalten. } \\
\text { Die Genehmigungen für Waffenexporte } \\
\text { werden fortan (seit dem 1. April 1998) vom } \\
\text { seco, , nach Absprache mit dem EDA, dem } \\
\text { VBS und dem UVEK erteilt. }\end{array}$ \\
\hline $\begin{array}{l}\text { Die Wohlfahrt durch } \\
\text { Armutsbekämpfung und } \\
\text { durch Erleichterung des } \\
\text { Zugangs zu den Märkten } \\
\text { des Nordens für die Produk- } \\
\text { te aus den Ent- } \\
\text { wicklungslåndern zu för- } \\
\text { dern. }\end{array}$ & $\begin{array}{l}\text { Tarifäre und nichttaritäre } \\
\text { Hindernisse für Einfuhren } \\
\text { von landwirtschaftlichen } \\
\text { Nahrungsmitteln aus } \\
\text { dem Ausland in die } \\
\text { Schweiz. }\end{array}$ & $\begin{array}{l}\text { ㄷ JSDW 1998, S. 286-2907 > } \\
\text { G JSDW 1999, S. 213-214 }\end{array}$ & $\begin{array}{l}\text { Verlängerung des Bundes- } \\
\text { beschlusses über Zollpră- } \\
\text { ferenzen für Entwicklungs- } \\
\text { länder um zehn Jahre - } \\
9.1996 \text {. } \\
\text { Revision der Verordnung } \\
\text { zur Festlegung der Prăfe- } \\
\text { renzzollansătze - } \\
\text { 1.3.1997 }\end{array}$ & $\begin{array}{l}\text { Die Zollpräferenzen wurden den am weite- } \\
\text { sten fortgeschrittenen Entwicklungsländern } \\
\text { entzogen, ohne die Zollpräferenzen für die } \\
\text { am wenigsten entwickelten Länder genü- } \\
\text { gend zu verbessern. }\end{array}$ \\
\hline
\end{tabular}

\begin{tabular}{|c|c|c|c|c|}
\hline $\begin{array}{l}\text { Die Kapitalflucht aus den } \\
\text { Entwicklungsländern zu ver- } \\
\text { hüten. }\end{array}$ & $\begin{array}{l}\text { Die Interessen des } \\
\text { Finanzplatzes Schweiz, } \\
\text { der einen Teil dieses } \\
\text { Kapitals aufnimmt. }\end{array}$ & & $\begin{array}{l}\text { Neues Geldwäscherei- } \\
\text { gesetz (GwG) - 1.4.1998. } \\
\text { g Jsow 1999. S. 180-190 }\end{array}$ & $\begin{array}{l}\text { त Verbesserung der Geldwäscherei- } \\
\text { bekämpfung durch Ausdehnung des Gest- } \\
\text { zes auf alle Finanzintermediäre. }\end{array}$ \\
\hline $\begin{array}{l}\text { Die Rechtshilfeverfahren } z \mathrm{u} \\
\text { beschleunigen. }\end{array}$ & $\begin{array}{l}\text { Das schweizerische Bank- } \\
\text { geheimnis und das } \\
\text { Berufsgeheimnis. }\end{array}$ & & $\begin{array}{l}\text { Revision des Gesetzes } \\
\text { von } 1996 \text { über internatio- } \\
\text { nale Rechtshilfe in Straf- } \\
\text { sachen - 1.2.1997 } \\
\text { a JSDW 1997. S. 101-102 }\end{array}$ & $\begin{array}{l}\rightarrow \text { Die Rechtshilfeverfahren sind weiter- }- \text { langsam. Die Gesetze wurden zwar } \\
\text { hin langsal } \\
\text { geändert, jedoch bleiben die menschlichen } \\
\text { und finanziellen Mittel zur Durchführung } \\
\text { von Untersuchungen unzureichend. }\end{array}$ \\
\hline $\begin{array}{l}\text { Die Korruption zu bekämp- } \\
\text { fen. }\end{array}$ & $\begin{array}{l}\text { Die Abzugsfähigkeit von } \\
\text { Bestechungsgeldern im } \\
\text { Ausland und die Unmög- } \\
\text { lichkeit, eine Person zu } \\
\text { verfolgen, die einen aus- } \\
\text { ländische Beamten be- } \\
\text { sticht (bis 1999). }\end{array}$ & $\begin{array}{l}\text { Korruptionsbekämpfung } \\
\text { - Leitlinien (Zusammen- } \\
\text { fassung der DEZA- } \\
\text { Arbeitsgruppe "Korrup- } \\
\text { tion». 25.8.1998). }\end{array}$ & $\begin{array}{l}\text { Revision des Strafgesetz- } \\
\text { buches zur Bestrafung } \\
\text { der Bestechung von } \\
\text { Beamten im Ausland - } \\
12.1999 \text {. } \\
\square \text { JSDW 2000, Abschnitt } 5.2 \text {. }\end{array}$ & $\begin{array}{l}\text { त Bestrafung der Bestechung ausländi- } \\
\text { scher Beamter und Abschaffung der steuer- } \\
\text { rechtlichen Abzugsfähigkeit von Beste- } \\
\text { chungsgeldern. }\end{array}$ \\
\hline $\begin{array}{l}\text { Globale Umweltpolitik und } \\
\text { schweizerische Beteiligung } \\
\text { an den multilateralen Platt- } \\
\text { formen. }\end{array}$ & $\begin{array}{l}\text { Nationale Umweltpolitik. } \\
\text { Beibehaltung des Kon- } \\
\text { summodells in der } \\
\text { Schweiz. }\end{array}$ & $\begin{array}{l}\text { "Nachhaltige Entwick- } \\
\text { lung in der Schweiz- } \\
\text { Strategie des Bundes- } \\
\text { rates". } \\
\text { JSDW 1998، S. 191-196 }\end{array}$ & $\begin{array}{l}\text { Ratifikation der multilate- } \\
\text { ralen Umweltkonven- } \\
\text { tionen durch die Schweiz } \\
\text { (Konventionen über biolo- } \\
\text { gische Vielfalt, Klima- } \\
\text { änderungen, Wüstenbil- } \\
\text { dung, Sonderabfälle). }\end{array}$ & 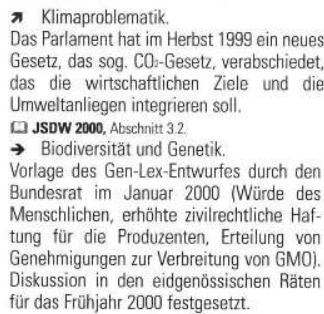 \\
\hline
\end{tabular}

\begin{tabular}{|c|c|c|c|c|}
\hline \multicolumn{2}{|c|}{ BEISPIELE } & \multicolumn{2}{|c|}{$\begin{array}{l}\text { IN VoRBEREITUNG BEFINDLICHE } \\
\text { ODER VERABSCHIEDETE MASSNAHMEN }\end{array}$} & \multirow{2}{*}{ 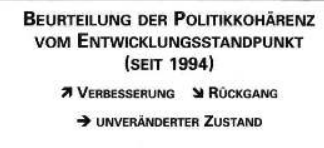 } \\
\hline $\begin{array}{l}\text { DAS LEITBILD NoRd-Sūo } \\
\text { ZIELT DARAUF AB: }\end{array}$ & $\begin{array}{l}\text { DAS LETBBIL NORD-Süd } \\
\text { KANN KONFUKTE IN FOLGENDEN } \\
\text { BERECHEN DEUTUCH MACHEN: }\end{array}$ & $\begin{array}{l}\text { AUF BUNDESVERWALIUNGS- } \\
\text { EBENE GETROFFENE MASS- } \\
\text { NAHMEN }\end{array}$ & $\begin{array}{l}\text { GesetzGeBUnGS- } \\
\text { MASSNAHMEN }\end{array}$ & \\
\hline $\begin{array}{l}\text { Die Menschenrechte in den } \\
\text { Entwicklungsländern zu för- } \\
\text { dern. }\end{array}$ & $\begin{array}{l}\text { Die kurzfristigen Interes- } \\
\text { sen der Schweiz in den } \\
\text { Entwicklungsländern. } \\
\text { Zum Beispiel: Erteilung } \\
\text { von Exportrisikogarantien } \\
\text { (ERG). }\end{array}$ & $\begin{array}{l}\text { Förderung der Menschen- } \\
\text { rechte und Entwick- } \\
\text { lungszusammearbeit - } \\
\text { Leitlinien } \\
\text { (Zusammenfassung der } \\
\text { transdepartementalen } \\
\text { A r b e e i } \mathrm{s} g \text { r u p p e } \\
\text { "Menschenrechte und } \\
\text { Rechtsstaatlichkeit ", } \\
\text { 1997). } \\
\text { Rechtsstaatlichkeitskon- } \\
\text { zept - Bedeutung in der } \\
\text { Entwicklungszusammen- } \\
\text { arbeit } \\
\text { (Zusammenfassung der } \\
\text { transdepartementalen } \\
\text { A r b e i } \mathrm{s} g \text { r u p p e } \\
\text { «Menschenrechte und } \\
\text { Rechtsstaatlichkeit ", } \\
\text { 10.1998). }\end{array}$ & & $\begin{array}{l}\text { ע Bei der Erteilung einiger Exportrisiko- } \\
\text { garantien haben die schweizerischen Inter- } \\
\text { essen (Exporttörderung) über wirtschaftliche } \\
\text { und soziale Erwägungen überwogen, } \\
\text { Beispiel: } \\
\text { ERG-Gewährung für die Projekte des Drei- } \\
\text { Schluchten-Staudamms (China) und des } \\
\text { llisu-Staudamms (Türkei). } \\
\text { D Jsow 1997, S. 129-130 } \\
\text { G JSDW 1998, S. 282-284 }\end{array}$ \\
\hline
\end{tabular}




\begin{tabular}{|c|c|c|c|}
\hline $\begin{array}{l}\text { Die Rechte der indigenen } \\
\text { Völker zu fördern. }\end{array}$ & $\begin{array}{l}\text { Schweizerische Wirt- } \\
\text { schaftsinteressen. } \\
\text { Beispiel 1. } \\
\text { Die TRIPS-Abkommen der } \\
\text { WTO, die von der } \\
\text { Schweiz unterstützt wer- } \\
\text { den, um die Interessen } \\
\text { der schweizerischen Un- } \\
\text { ternehmen zu wahren. } \\
\\
\text { Beispie/ 2. } \\
\text { Verteidigung oder Förde- } \\
\text { rung der Schweiz als } \\
\text { internationaler Kunst- } \\
\text { markt. }\end{array}$ & $\begin{array}{l}\text { Das Engagement der } \\
\text { Schweiz für die indige- } \\
\text { nen Völker (Zusammen- } \\
\text { fassung des Gedanken- } \\
\text { austausches zwischen } \\
\text { EDA und NRO, } 1996 \\
\text {-1998). }\end{array}$ & 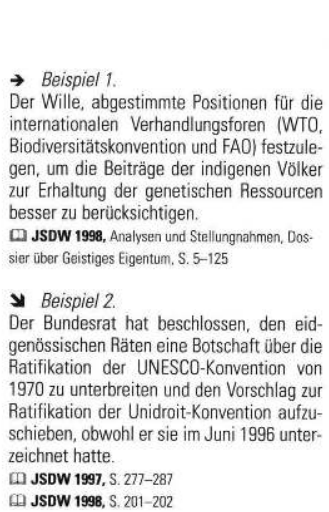 \\
\hline
\end{tabular}

Tabelle nach : Greminger Thomas, „La cohérence des politiques - un premier bilan de la mise en oeuvre des Lignes directrices Nord-Sud de la Suisse", in Fino Daniel (dir), Impasses et promesses..., S. 152.

\section{BIBLIOGRAPHY}

Beratende Kommission für internationale Entwicklungszusammenarbeit, Migration und Entwicklungszusammenarbeit : Synergien und Zielkonflikte, Thesenpapier, 1996.

Interdepartementaler Ausschuss Rio (IDA-Rio), Nachhaltige Entwicklung in der Schweiz, Stand der Realisierung, Bern, 1997, 58 S.

Commission mondiale sur l'environnement et le développement. Notre avenir à tous, Editions du Fleuve, Montréal, 1988, 454 S.

Schweizerischer Bundesrat, Bericht des Bundesrates über die Nord-Süd-Beziehungen der Schweiz in den 90er Jahren. „Leitbild Nord-Süd”, vom 7. März 1994, DEZA, Bern, 1994, 28 S.

Schweizerischer Bundesrat, Bericht über die Aussenpolitik der Schweiz in den 90er Jahren, vom 29. November 1993, Botschaft 93.098, 1993.

DDC, Plan d'action de la DDC pour la mise en œuvre des Lignes directrices Nord-Sud 1995-1998, Berne, 1995, 38 S. ( Internet-Version ).

DEZA, Förderung der Menschenrechte und Entwicklungszusammenarbeit. Leitlinien, Bern, 1998, $24 \mathrm{~S}$.

DEZA, Strategisches Controlling/Politik und Forschung, Systemik der Planungsinstrumente in der DEZA. Arbeitsinstrument der DEZA 6/99, Bern, 1999.

J. Forster, „The Coherence of Policies towards Developing countries : The Case of Switzerland”, in J. Forster and O. Stokke (dir.), Policy Coherence in Development Co-operation, Frank Cass, EADI Book Series 22, London, 1999, S. 295-322.

T. Greminger, „La cohérence des politiques - un premier bilan de la mise en œuvre des Lignes directrices Nord-Sud de la Suisse", in D. Fino (dir.), Impasses et promesses. L'ambiguité de la 
coopération au développement, PUF/IUED, coll. Enjeux, Nouveaux Cahiers de l'IUED, $\mathrm{n}^{\circ} 4$, Paris, Genève, 1996, S. 141-154.

P. Hoebink, „Cohérence and Development Policy : The Case of the European Union”, in J. Forster and O. Stokke (dir.), Policy Cohérence in Development Co-operation, Frank Cass, EADI Book Series 22, London, 1999, S. 323-345.

P. Maurer, „Überlegungen zum Thema Kohärenz”, in Jahrbuch Schweiz - Dritte Welt 1995, IUED, Genf, 1995, S. 162-166.

OCDE, Série des examens en matière de coopération pour le développement : Suisse. $1996, \mathrm{n}^{\circ} 20$, Comité d'aide au développement, Paris, 1997, 63 S.

BUWAL, Strategie des Amtes, (Version Februar 1999), Bern, 1999, 33 S.

\section{NOTES}

1. Bericht über die Aussenpolitik der Schweiz in den 90er Jahren, vom 20. November 1993, und Bericht des Bundesrates Über die Nord-Süd-Beziehungen der Schweiz in den 90er Jahren. „Leitbild Nord-Süd”, vom 7. März 1994.

2. Unsere Untersuchung befasst sich weder mit dem Einfluss des Leitbilds Nord-Süd auf die Tätigkeit des schweizerischen Privatsektors - ob Wirtschaftssektor oder Vereinswesen - noch mit seinem Einfluss auf die Positionen der Schweiz in den multilateralen Institutionen.

3. DEZA : Jean-François Giovannini, Stellvertretender Direktor; Marco Rossi, Chef der Sektion Politik und Forschung.

seco : Nicolas Imboden, Direktor des Leistungszentrums Entwicklung und Transition.

BUWAL : Philippe Roch, Direktor.

BFF, Jean-Daniel Gerber, Direktor ; Jörg Frieden, Chef des Bereichs der Direktion Finanzen und Soziales.

PAIV : Jean-Daniel Vigny, ministre, Christoph Pappa, diplomatischer Mitarbeiter.

4. Für mehr Einzelheiten zu diesem Thema siehe den Artikel von J. Forster im Dossier des vorliegenden Jahrbuchs.

5. Für eine Beschreibung der Entstehungsgeschichte des Leitbilds Nord-Süd verweisen wir den Leser/die Leserin auf den Text von T. Greminger, „La cohérence des politiques - un premier bilan de la mise en œuvre des Lignes directrices Nord-Sud de la Suisse", in D. Fino (dir.), Impasses et promesses. L'ambiguité de la coopération au développement, PUF/IUED, coll. Enjeux, Nouveaux Cahiers de l'IUED, nº 4, Paris/Genève, 1996, S. 144-146.

6. Der Bericht über die Aussenpolitik der Schweiz in den 90er Jahren, der den Bezugsrahmen des LBNS bildet, enthält fünf Ziele. Im LBNS wurden die beiden ersten Ziele zu einem einzigen Ziel zusammengefasst.

7. Die Definition dieser Ebenen ist dem folgenden Artikel von J. Forster entnommen : „The Coherence of Policies towards Developing Countries : The Case of Switzerland", in J. Forster and O. Stokke (dir.), Policy Cohérence in Development Co-operation, Frank Cass, EADI Book Series 22, London, 1999, S. 295-322.

8. Ibid, S. 308.

9. Laut P. Hoebink, „Cohérence and Development Policy: The Case of the European Union”, in J. Forster and O. Stokke (dir.), Policy Cohérence in Development Co-operation, S. 335.

10. Commission mondiale sur l'environnement et le développement, Notre avenir à tous, Editions du Fleuve, Montréal, 1988, S. 51.

Definition der Weltkommission für Umwelt und Entwicklung im Bericht des 
Interdepartementalen Ausschusses Rio (IDARio), Nachhaltige Entwicklung in der Schweiz, Bern, Februar 1996, S. 9.

11. Commission mondiale sur l'environnement..., Ibid.

12. Bundesrat, Bericht des Bundesrates über die Nord-Süd-Beziehungen..., S. 12.

13. Ibid.

14. Ibid.

15. Ibid., S. 11.

16. DDC, Plan d'action de la DDC pour la mise en cuvre des Lignes directrices Nord-Sud 1995-1998, Bern, 1995, 38 S. (Aktionsplan zum Leitbild Nord-Süd 1995-1998).

17. Aus vielleicht verständlichen Gründen besteht zu Umweltfragen in der Bundesverwaltung ein ziemlich breiter Konsens.

18. DDC, Plan d'action de la DDC..., S. 37.

19. Ibid., S. 24.

20. DEZA, Strategisches Controlling/Politik und Forschung, Systemik der Planungsinstrumente in der DEZA. Arbeitsinstrument der DEZA 6/99, Bern, 1999, Tabelle 1: „Systemik der Planungsinstrumente und Führungsebenen".

21. Bundesrat, Bericht des Bundesrates über die Nord-Süd-Beziehungen..., S. 12.

22. Das seco ist aus der Zusammenlegung des BAWI und des Bundesamtes für Wirtschaft und Arbeit im Juli 1999 hervorgegangen.

23. Das seco umfasst acht „Leistungszentren”, darunter das Leistungszentrum Entwicklung und Transition, das sich speziell mit den Beziehungen mit dem Süden und Osten befasst. Die Organisationsstruktur des seco ist auf der Internet-Seite http://www.seco.admin.ch - „Wer wir sind" einzusehen.

24. BUWAL, Strategie des Amtes (Version Februar 1999), Bern, 1999, S. 1.

Frz. Text: Dans sa stratégie, l'OFEFP met en avant son röle moteur dans „'intégration du pilier environnemental du développement durable dans les politiques nationales et internationales" de la Suisse.

25. Indem man, mutatis mutandis, der Theorie der komparativen Vorteile folgt.

26. Beratende Kommission für internationale Entwicklungszusammenarbeit, Migration und Entwicklungszusammenarbeit : Synergien und Zielkonflikte, Thesenpapier, 1996.

27. DEZA, Förderung der Menschenrechte und Entwicklungszusammenarbeit, Leitlinien, Bern, 1998, 24 S.

28. Peter Maurer, „Überlegungen zum Thema Kohärenz”, in Jahrbuch Schweiz-Dritte Welt 1995, IUED, Genf. 1995, S. 165.

29. Ibid., S. 166.

30. Interdepartementaler Ausschuss Rio (IDARio), Nachhaltige Entwicklung in der Schweiz. Stand der Realisierung, Bern, 1997, S. 3.

\section{AUTHORS}

\section{XAVIER TSCHUMI}

Lie. ès se. écon., Universität Genf, Student am IUED, Genf

\section{CATHERINE SCHÜMPERLI YOUNOSSIAN}

Forschungsbeauftragte am IUED, Genf. 